\title{
A 52-Week Trial Comparing Briakinumab with Methotrexate in Patients with Psoriasis
}

\author{
Kristian Reich, M.D., Richard G. Langley, M.D., Kim A. Papp, M.D., \\ Jean-Paul Ortonne, M.D., Kristina Unnebrink, Ph.D., Martin Kaul, M.D., \\ and Joaquin M. Valdes, M.D.
}

From the Dermatologikum Hamburg, Hamburg (K.R.), Georg-August-University, Göttingen (K.R.), and Abbott, Ludwigshafen (K.U., M.K.) — all in Germany; Dalhousie University, Halifax, NS (R.G.L.), Probity Medical Research, Waterloo, ON (K.A.P.), and University of Western Ontario, London (K.A.P.) - all in Canada; Hôpital de L'Archet, Nice, France (J.-P.O.); and Abbott Laboratories, Abbott Park, IL (J.M.V.). Address reprint requests to Dr. Reich at Dermatologikum Hamburg, Stephansplatz 5, Hamburg 20354, Germany, or at kreich@dermatologikum.de.

N EnglJ Med 2011;365:1586-96. Copyright (C) 2011 Massachusetts Medical Society.

\section{A BSTRACT}

\section{BACKGROUND}

Briakinumab is a monoclonal antibody against the p40 molecule shared by interleukin-12 and interleukin-23, which is overexpressed in psoriatic skin lesions. We assessed the efficacy and safety of briakinumab as compared with methotrexate in patients with psoriasis.

\section{METHODS}

In this 52-week trial, we randomly assigned 317 patients with moderate-to-severe psoriasis to briakinumab, at a dose of $200 \mathrm{mg}$ at weeks 0 and 4 and $100 \mathrm{mg}$ at week 8 and every 4 weeks thereafter (154 patients), or methotrexate, at a dose of 5 to $25 \mathrm{mg}$ weekly (163 patients). The primary end points were the percentages of patients with at least 75\% improvement in the score on the psoriasis area-and-severity index (PASI) at weeks 24 and 52 and a score on the physician's global assessment of 0 (clear; i.e., no apparent disease) or 1 (minimal disease) at weeks 24 and 52. A total of 248 patients were enrolled in an ongoing 160-week open-label continuation study.

\section{RESULTS}

At week 24 , a total of $81.8 \%$ of the patients in the briakinumab group versus $39.9 \%$ in the methotrexate group had at least $75 \%$ improvement in the PASI score, and $80.5 \%$ versus $34.4 \%$ had a score of 0 or 1 on the physician's global assessment. The corresponding percentages at week 52 were $66.2 \%$ versus $23.9 \%$ with at least a $75 \%$ improvement in the PASI score and $63.0 \%$ versus $20.2 \%$ with a score of 0 or 1 on the physician's global assessment ( $\mathrm{P}<0.001$ for all comparisons). During the 52 -week study, serious adverse events occurred in $9.1 \%$ of the patients in the briakinumab group (12.9 events per 100 patient-years) and in 6.1\% in the methotrexate group (10.6 events per 100 patient-years). Serious infections occurred in $2.6 \%$ of the patients in the briakinumab group (4.1 events per 100 patient-years) and in $1.8 \%$ in the methotrexate group ( 2.7 events per 100 patient-years); cancers occurred in $1.9 \%$ ( 2.0 events per 100 patient-years) versus $0 \%$.

\section{CONCLUSIONS}

Briakinumab showed higher efficacy than methotrexate in patients with moderateto-severe psoriasis. Serious infections and cancers occurred more frequently with briakinumab, but the differences were not significant. (Funded by Abbott Laboratories; ClinicalTrials.gov number, NCT00679731.) 
P SORIASIS IS A CHRONIC SKIN DISEASE THAT is characterized by T-cell-mediated systemic inflammation ${ }^{1,2}$ and is associated with considerable impairment in health-related quality of life and physical and mental functioning. ${ }^{3,4}$ Interleukin-12 and interleukin-23 play important roles in the pathogenesis of psoriasis., ${ }^{1,2}$ Interleukin-12 is associated with the type 1 helper T-cell (Th1) phenotype, leading to production of interferon- $\gamma$; interleukin-23 fosters the type 17 helper T-cell (Th17) phenotype and the production of interleukin-17 and interleukin-22. The shared p40 subunit of interleukin-12 and interleukin-23 is overexpressed in psoriatic skin lesions ${ }^{5-7}$ and is linked to psoriasis in genetic studies. ${ }^{8-10}$ Ustekinumab (CNTO 1275, Centocor) ${ }^{11,12}$ and briakinumab (ABT-874, Abbott Laboratories) ${ }^{13,14}$ — monoclonal antibodies targeting the p40 molecule shared by interleukin-12 and interleukin-23 - have shown clinical efficacy in patients with psoriasis.

Although there is strong evidence supporting the efficacy of biologic therapy for the treatment of psoriasis, ${ }^{15}$ there is a need to compare the efficacy and safety of biologic agents with those of traditional systemic therapies such as methotrexate, which is the most commonly prescribed systemic therapy for psoriasis worldwide. ${ }^{16}$ We evaluated the efficacy and safety of 1 year of treatment with briakinumab as compared with methotrexate in patients with moderate-to-severe plaque psoriasis.

\section{METHODS}

\section{PATIENTS}

We conducted this phase 3, multicenter, randomized, double-blind trial from May 2008 through November 2009 at 43 sites in Europe and Canada. Patients 18 years of age or older were eligible to participate if they had had psoriasis, diagnosed clinically, for at least 6 months and stable plaque psoriasis for at least 2 months; were candidates for systemic therapy or phototherapy; had at least $10 \%$ of their body-surface area affected by psoriasis; and had a score on the physician's global assessment of 3 or higher and a score on the psoriasis area-andseverity index (PASI) of 12 or higher at baseline. (Scores on the physician's global assessment range from 0 to 5 , with scores of 2, 3, 4, and 5 indicating mild, moderate, severe, and very severe disease, respectively; PASI scores range from 0 to 72 , with higher scores indicating more severe disease.) For additional details, see the Supplementary Appendix, available with the full text of this article at NEJM.org. Written informed consent was obtained from all patients.

\section{STUDY OVERSIGHT}

Abbott Laboratories sponsored the study, which was designed jointly by academic advisers and Abbott personnel. Abbott conducted the data analyses, and all the authors had full access to the data; the first author vouches for the completeness and accuracy of the data presented and of the data analyses. The first draft of the manuscript was written by a medical writer employed by Abbott, with input from all the authors. All the authors reviewed and provided feedback on all subsequent versions of the manuscript and made the decision to submit it for publication. All the authors signed confidentiality disclosures with the sponsor regarding these data.

The study protocol was approved by an independent ethics committee or institutional review board at each participating site. The protocol, including the statistical analysis plan, is available at NEJM.org. All the authors vouch for the fidelity of the study to the protocol.

\section{PROCEDURES}

At baseline (week 0), patients were randomly assigned, in a 1:1 ratio, to receive briakinumab, administered subcutaneously at a dose of $200 \mathrm{mg}$ at weeks 0 and 4 and 100 mg every 4 weeks from week 8 through week 48 , or methotrexate, administered orally at a dose of 5 to $25 \mathrm{mg}$ per week, plus folate, administered orally at a dose of $5 \mathrm{mg}$ per week, from week 0 through week 51 (Fig. 1). Patients in the methotrexate group received $5 \mathrm{mg}$ of methotrexate at week $0,10 \mathrm{mg}$ at week 1 , and $15 \mathrm{mg}$ per week from week 2 through week 9. The dose of methotrexate was increased to $20 \mathrm{mg}$ per week at week 10 and to $25 \mathrm{mg}$ per week at week 16 in patients who did not meet the criterion of at least a $75 \%$ improvement in the PASI score or of a score on the physician's global assessment of 0 or 1 . Neither the patients nor the investigators were aware of the treatment assignments. Placebo capsules that looked identical to the methotrexate capsules and folate or placebo injections to match briakinumab injections were administered to maintain the concealment of the treatment assignments.

Treatment success was defined as both an im- 


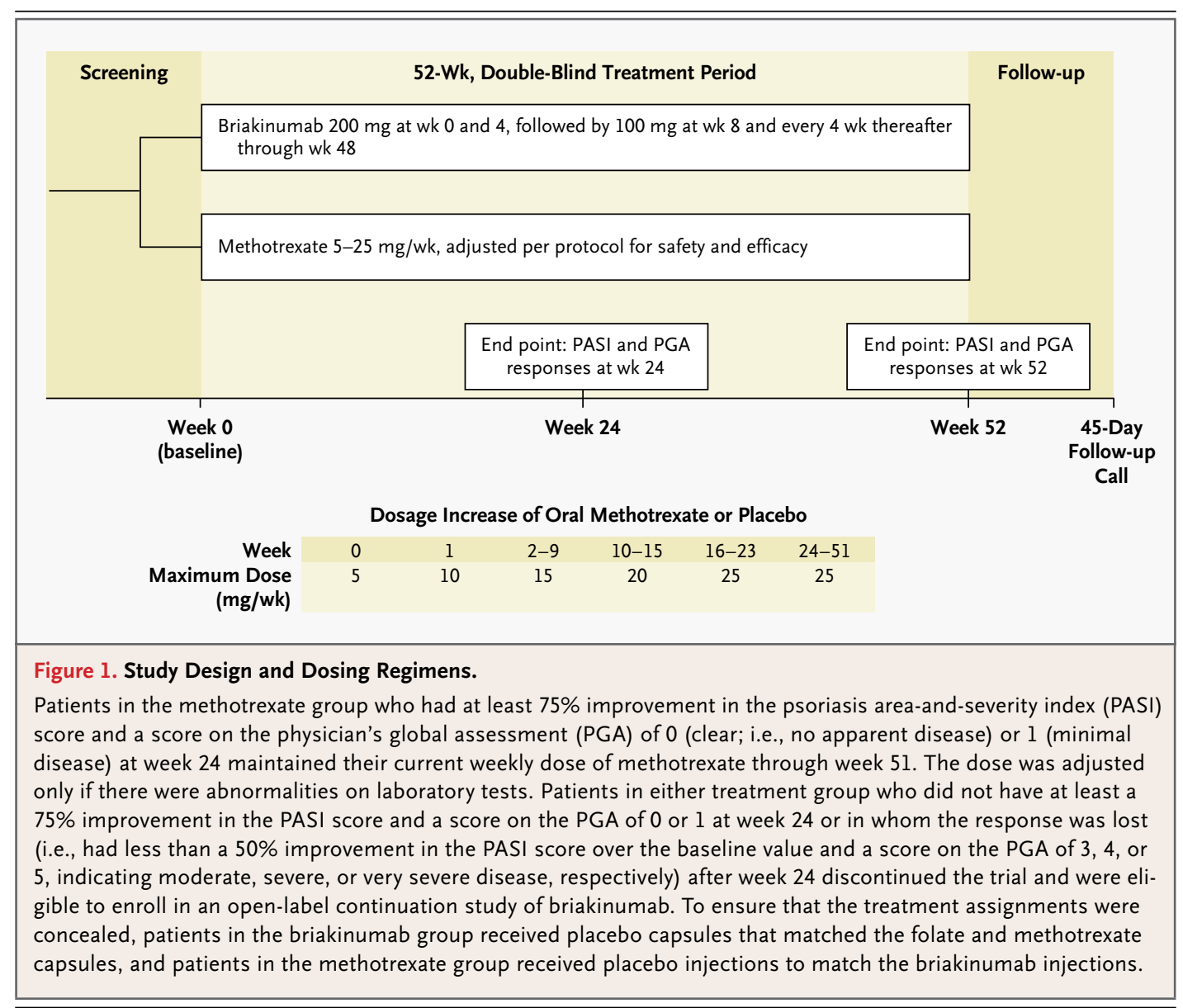

provement of at least $75 \%$ from baseline in the PASI score and a score on the physician's global assessment of 0 (clear; i.e., no apparent disease) or 1 (minimal disease) at week 24. Patients in the methotrexate group who met the criteria for treatment success continued to take their current weekly dose of methotrexate for the remainder of the study. At any time during the study, the safety assessor could reduce or withhold the dose of methotrexate if warranted by the development of symptoms reported by the patient, adverse events, or laboratory abnormalities or by the results of a physical examination. Patients in either treatment group who did not meet the criteria for treatment success or in whom the response was lost (i.e., had $<50 \%$ improvement in the PASI score over the baseline value and a score on the physician's global assessment of $\geq 3$ ) after week 24 discontinued the trial and were eligible to enroll in an ongoing open-label continuation study in which all patients receive briakinumab.

\section{STUDY END POINTS}

The primary efficacy end points were the percentages of patients with at least $75 \%$ improvement in the PASI score at week 24 , a score on the physician's global assessment of 0 or $1^{17}$ at week 24, at least $75 \%$ improvement in the PASI score at week 52, and a score on the physician's global assessment of 0 or 1 at week 52 .

Secondary efficacy end points included the median time to reach the end point of at least $75 \%$ improvement in the PASI score; rates of improvement of at least $50 \%, 75 \%, 90 \%$, and $100 \%$ in the PASI score as assessed at all study visits through week 52; mean percentage improvements in PASI scores from baseline; the change from baseline in the score on the Nail Psoriasis Severity Index (in which scores range from 0 , indicating no nail psoriasis, to 8 , indicating psoriasis in $100 \%$ of the nail ${ }^{18}$ ) for the nail most affected by psoriasis at baseline; and assessments of patients on the basis of the Dermatology Life Quality Index (DLQI), 
which measures the effect of dermatologic diseases on the quality of life (on a scale from 0 to 30 , with lower scores indicating a lesser effect on health-related quality of life). ${ }^{19}$ The DLQI assessments included the percentage of patients with a DLQI score of 0 or 1 (indicating no effect of psoriasis), ${ }^{20}$ the percentage of patients with a decrease of at least 5 points (a clinically meaningful reduction), ${ }^{21}$ and mean reductions in DLQI scores from baseline.

Adverse events, laboratory data, and vital signs were assessed throughout the study. Treatmentemergent adverse events were defined as those occurring at the time the first dose of study drug was administered or any time thereafter, up to 45 days after the last dose was administered (see the Supplementary Appendix for methods related to the open-label continuation study). A follow-up call was made 45 days after the last dose of the study drug was administered.

\section{STATISTICAL ANALYSIS}

According to the original study plan, we estimated that with approximately 250 patients undergoing randomization, the study would have $90 \%$ power to show a greater response with briakinumab than with methotrexate (i.e., at least $75 \%$ improvement in the PASI score at week 24 in $70 \%$ of the briakinumab group as compared with $50 \%$ of the methotrexate group), at a two-sided significance level of $5 \%$ with the use of a chi-square test. However, owing to difficulties in obtaining accurate reports of the enrollment numbers in a timely manner, more than 250 patients were enrolled. With an actual enrollment number of 317 patients, the power increased from $90 \%$ to $95 \%$.

The efficacy analyses were performed on data from the intention-to-treat population (all patients who underwent randomization). All primary efficacy comparisons were performed with the use of a two-sided Cochran-Mantel-Haenszel test with adjustment for country, at an alpha level of 0.05 . We maintained the type I error rate at 0.05 by adhering to the a priori defined order of statistical hypotheses. The primary end points were ordered a priori as follows: at least $75 \%$ improvement in the PASI score at week 24 , a score on the physician's global assessment of 0 or 1 at week 24 , at least $75 \%$ improvement in the PASI score at week 52, and a score on the physician's global assessment of 0 or 1 at week 52 . Thus, the superiority of briakinumab over methotrexate could be claimed for an end point only if briakinumab had been shown to be superior for all previous end points. Any patient with a missing score on the PASI or physician's global assessment at a visit was considered not to have had a response at that visit (imputation of no response). The last-observation-carried-forward method was used for a sensitivity analysis.

For other categorical variables, the chi-square test or Fisher's exact test (if the expected cell size was $<5$ ) was used to evaluate differences between the treatment groups. Missing values for categorical secondary end points were handled in the same way as missing values for primary end points. The median times to reaching the end points of at least $75 \%$ improvement in the PASI score and a score on the physician's global assessment of 0 or 1 were calculated with the use of the Kaplan-Meier method. The between-group difference was tested with the use of the log-rank test. Data from patients who did not have treatment success on or before week 52 were included in the analysis as censored observations, with the observation time calculated as the interval from week 0 (baseline) to the date of the last assessment of the PASI score or the last physician's global assessment.

Differences between treatment groups in the change from baseline in scores on the PASI and DLQI and in other continuous variables were analyzed with the use of analysis of covariance, with baseline value in the model. For the percentage change in the score on the Nail Psoriasis Severity Index, only patients with nonzero baseline scores were included in the analysis. Wilcoxon two-sample tests were used to compare the between-group difference in the change. For these assessments, the last-observation-carried-forward method was used in the case of missing data, and analysis as observed (i.e., no imputation) was performed as a sensitivity analysis.

The safety analyses were conducted on data from the safety population, which included all patients who received at least one dose of a study drug. Adverse events that occurred during both the 52-week trial and the open-label continuation study were summarized as the number and percentage of patients with treatment-emergent adverse events and as the number of events per 100 person-years. During the 52-week trial, the rate of adverse events was compared between treatment groups with the use of Fisher's exact test. 


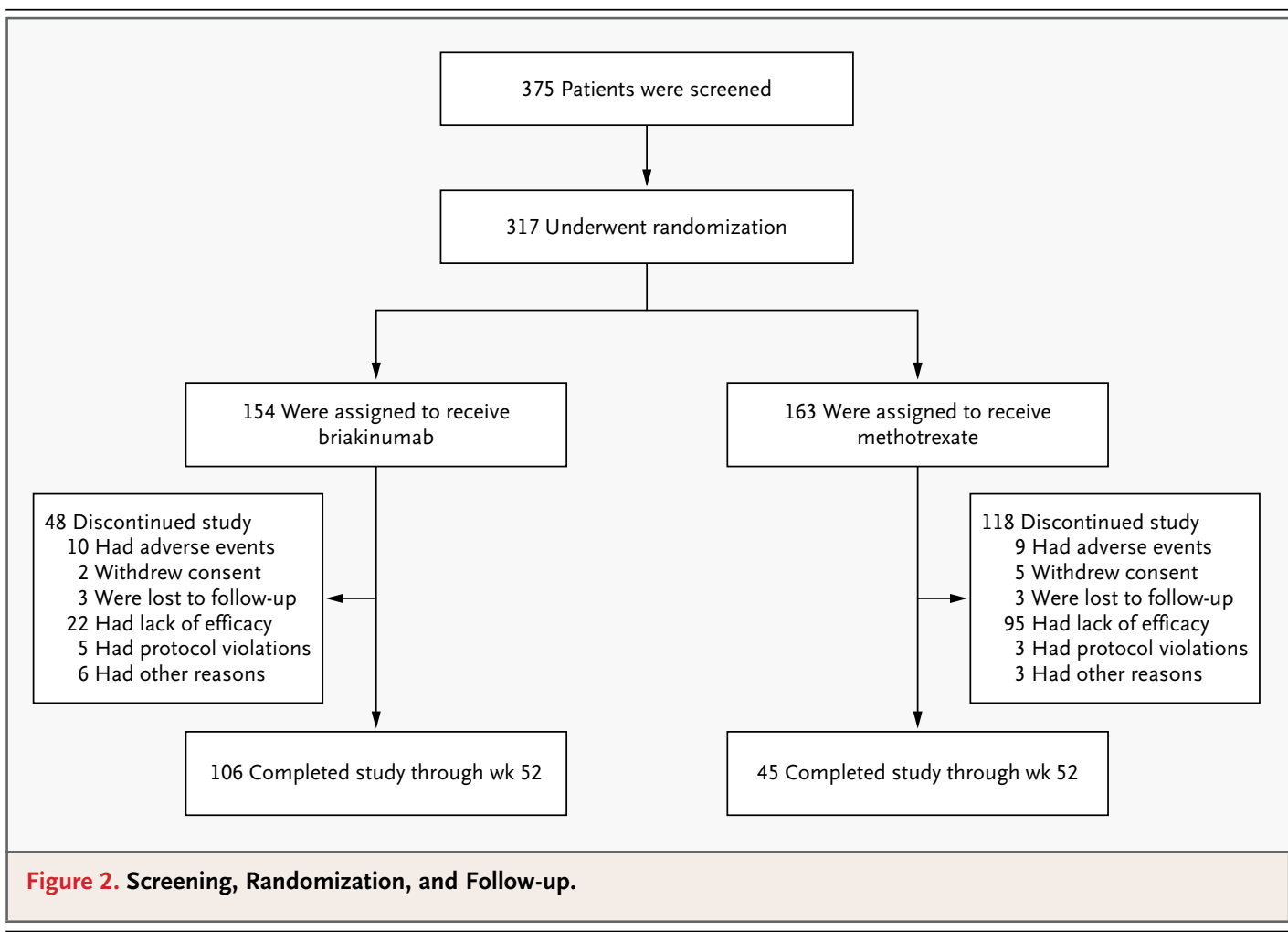

RESULTS

\section{PATIENTS}

A total of 317 patients met the inclusion criteria and were randomly assigned to briakinumab (154) or methotrexate (163) (intention-to-treat population) (Fig. 2). Baseline demographic and clinical characteristics and disease severity were generally similar in the two groups (Table 1); no significant differences were seen, with the exception of the overall score on the physician's global assessment $(\mathrm{P}=0.04)$. Per protocol, 17 patients in the methotrexate group required an increase in the dose to $20 \mathrm{mg}$ per week at week 10 , and 100 patients in that group required an increase in the dose to $25 \mathrm{mg}$ per week at week 16.

\section{EFFICACY}

PASI

Significantly more patients in the briakinumab group than in the methotrexate group met the criterion for the primary end point of at least $75 \%$ improvement in the PASI score, both at week $24(81.8 \%$ vs. $39.9 \%)$ and at week $52(66.2 \%$ vs. $23.9 \%)(\mathrm{P}<0.001$ for the comparison at both time points). At week 12 , a total of $76.6 \%$ of the patients in the briakinumab group, as compared with $36.2 \%$ in the methotrexate group, had at least $75 \%$ improvement in the PASI score $(\mathrm{P}<0.001)$. The median time to an improvement of at least $75 \%$ in the PASI score was 56 days in the briakinumab group, as compared with 140 days in the methotrexate group $(\mathrm{P}<0.001)$. The percentage of patients who had at least $75 \%$ improvement in the PASI score was significantly greater in the briakinumab group than in the methotrexate group by week 4 and at all time points through week 52 (Fig. 3A). (The numbers of patients available for follow-up according to study visit are shown in Table 1 in the Supplementary Appendix.) Significant between-group differences in the percentage of patients with at least $50 \%$ improvement in the PASI score were apparent beginning at week 2 (Fig. 1A in the Supplementary Appendix), and significant differences in the percentages of patients with at least $90 \%$ improvement and with $100 \%$ improvement in the PASI score were apparent at week 8 (Fig. 3B). The mean percentage improvement from baseline in PASI scores increased over time in both treatment groups but was significantly greater in the briakinumab group at all time points (Fig. 1B in the Supplementary Appendix). 


\begin{tabular}{|c|c|c|}
\hline Characteristic & $\begin{array}{c}\text { Briakinumab } \\
(N=154)\end{array}$ & $\begin{array}{c}\text { Methotrexate } \\
(\mathrm{N}=163)\end{array}$ \\
\hline Age $-y r$ & $45.0 \pm 13.1$ & $43.1 \pm 12.9$ \\
\hline Male sex - no. (\%) & $111(72.1)$ & $111(68.1)$ \\
\hline White race - no. (\%) $†$ & $149(96.8)$ & $158(96.9)$ \\
\hline Weight $-\mathrm{kg}$ & $85.1 \pm 17.5$ & $82.0 \pm 18.6$ \\
\hline Duration of psoriasis - yr & $18.6 \pm 11.9$ & $19.1 \pm 11.3$ \\
\hline Body-surface area affected by psoriasis — \% & $26.1 \pm 16.7$ & $26.1 \pm 16.3$ \\
\hline History of psoriatic arthritis — no. (\%) & $25(16.2)$ & $28(17.2)$ \\
\hline PASI score & $18.4 \pm 6.7$ & $17.8 \pm 6.1$ \\
\hline \multicolumn{3}{|l|}{ NAPSI score』 } \\
\hline Overall & $27.7 \pm 16.4$ & $27.9 \pm 19.1$ \\
\hline Target fingernail & $4.8 \pm 2.0$ & $4.8 \pm 2.1$ \\
\hline \multicolumn{3}{|l|}{ PGA score - no. (\%) } \\
\hline $0-2$ & 0 & 0 \\
\hline 3 & $75(48.7)$ & $87(53.4)$ \\
\hline 4 & $65(42.2)$ & $72(44.2)$ \\
\hline 5 & $14(9.1)$ & $4(2.5)$ \\
\hline DLQI score\| & $11.1 \pm 6.6$ & $11.3 \pm 7.5$ \\
\hline \multicolumn{3}{|l|}{ Previous psoriasis treatment - no. (\%) } \\
\hline Topical therapy & $141(91.6)$ & $148(90.8)$ \\
\hline Phototherapy*** & $100(64.9)$ & $105(64.4)$ \\
\hline Systemic nonbiologic treatment & $77(50.0)$ & $89(54.6)$ \\
\hline Systemic biologic treatment & $24(15.6)$ & $34(20.9)$ \\
\hline Etanercept & $14(9.1)$ & $12(7.4)$ \\
\hline Infliximab & $9(5.8)$ & $11(6.7)$ \\
\hline Adalimumab & $5(3.2)$ & $7(4.3)$ \\
\hline Alefacept & $2(1.3)$ & $2(1.2)$ \\
\hline Efalizumab & $6(3.9)$ & $3(1.8)$ \\
\hline Other & $3(1.9)$ & $7(4.3)$ \\
\hline
\end{tabular}

* Plus-minus values are means \pm SD. None of the characteristics differed significantly between the two groups with the exception of the overall score on the physician's global assessment (PGA) $(P=0.04)$.

$\dagger$ Race was determined by the investigator.

Scores on the psoriasis area-and-severity index (PASI) range from 0 to 72, with higher scores indicating more severe disease.

$\int$ Scores on the Nail Psoriasis Severity Index (NAPSI) range from 0, indicating no nail psoriasis, to 8, indicating psoriasis in $100 \%$ of the nail. Overall scores are the sum of scores for each of the 10 fingernails. NAPSI scores were available for 115 patients in the briakinumab group and 108 in the methotrexate group.

If Scores on the PGA range from 0 to 5 , with 0 indicating no psoriasis, 1 minimal psoriasis, 2 mild psoriasis, 3 moderate psoriasis, 4 severe psoriasis, and 5 very severe psoriasis.

\| Scores on the Dermatology Life Quality Index (DQLI) range from 0 to 30, with lower scores indicating a lower effect on health-related quality of life. Scores on the DQLI were available for 154 patients in the briakinumab group and 162 in the methotrexate group.

$* *$ Phototherapy included psoralen and ultraviolet A (PUVA) and narrow-band ultraviolet B (UVB) therapies.

\section{Physician's Global Assessment}

Significantly more patients in the briakinumab group than in the methotrexate group met the criterion of a score of 0 or 1 on the physician's global assessment, both at week 24 (80.5\% vs. $34.4 \%)$ and at week 52 (63.0\% vs. $20.2 \%)(\mathrm{P}<0.001$ for the comparison at both time points). At week 12, a total of $68.2 \%$ of the patients in the briakinumab group, 


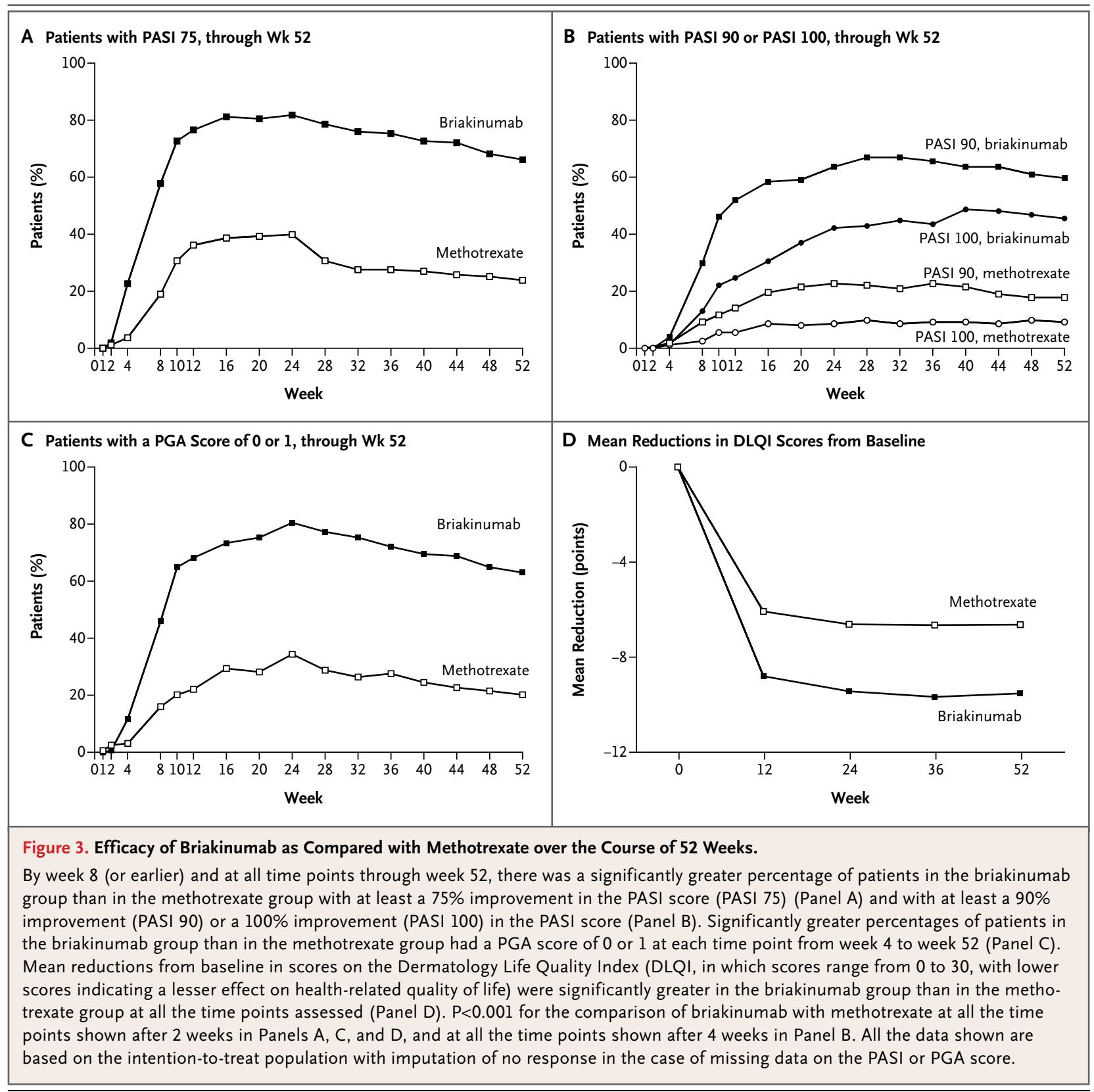

as compared with $22.1 \%$ in the methotrexate group, had a score of 0 or $1(\mathrm{P}<0.001)$. Significantly more patients in the briakinumab group than in the methotrexate group had a score of 0 (clear) at week 24 (46.1\% vs. $9.2 \%)$ and at week 52 (45.5\% vs. $9.8 \%$ ) $(\mathrm{P}<0.001$ for the comparison at both time points). The median time to a score of 0 or 1 on the physician's global assessment was 69 days in the briakinumab group, as compared with 171 days in the methotrexate group $(P<0.001)$. Significantly greater percentages of patients in the briakinumab group than in the methotrexate group had a score of 0 or 1 at each time point from week 4 to week 52 (Fig. 3C).

\section{Other Assessments}

Baseline scores on the Nail Psoriasis Severity Index were similar in the two treatment groups (Table 1). Mean scores for the target fingernail at baseline, week 24, and week 52 were 4.8, 2.1, and 1.2, respectively, in the briakinumab group, as compared with $4.8,3.0$, and 3.0, respectively, in the 
methotrexate group ( $\mathrm{P}<0.001$ for the change from baseline with briakinumab vs. methotrexate).

More patients in the briakinumab group than in the methotrexate group had a score of 0 or 1 on the DLQI, both at week 24 (70.8\% vs. 34.4\%) and at week 52 (61.7\% vs. $17.8 \%)(\mathrm{P}<0.001$ for the comparison at both time points). Similarly, significantly more patients in the briakinumab group than in the methotrexate group had a clinically meaningful reduction in the DLQI score, both at week 24 (66.2\% vs. $47.9 \%)$ and at week 52 (56.5\% vs. $18.4 \%)(\mathrm{P}<0.001$ for the comparison at both time points). Mean reductions in DLQI scores from baseline were significantly greater in the briakinumab group than in the methotrexate group at all the time points assessed (Fig. 3D).

For all efficacy variables, sensitivity analyses performed to account for missing data yielded similar results (data not shown).

\section{ADVERSE EVENTS}

During the 52-week trial, the most common treatment-emergent adverse events were nasopharyngitis, headache, diarrhea, arthralgia, and upper respiratory tract infection (Table 2). Diarrhea and adverse events related to the injection site occurred more frequently among patients receiving briakinumab than among those receiving methotrexate.

A total of 12 patients in the briakinumab group (7.8\%) discontinued the study owing to adverse events; in the case of 5 of these patients, the adverse events were serious (gastrointestinal hypomotility coincident with legionella infection, breast cancer, breast neoplasm [intraductal carcinoma], prostate cancer, and herpes zoster). A total of 10 patients in the methotrexate group (6.1\%) discontinued the study owing to adverse events; in the case of 5 of these patients, the adverse events were serious (increased hepatic enzyme levels and hepatitis, sacroiliitis, diverticulitis, erythrodermic psoriasis, and angioedema and urticaria).

A total of 14 patients in the briakinumab group $(9.1 \%)$ and 10 in the methotrexate group $(6.1 \%)$ had treatment-emergent serious adverse events; 7 patients had serious infections: 4 in the briakinumab group (one case of legionella infection with candidemia and septic shock, one case of osteomyelitis, one case of herpes zoster, and one case of tonsillitis) and 3 in the methotrexate group (two cases of diverticulitis and one case of drug-induced hepatitis). The incidence rate of serious infectious adverse events was 4.1 per 100 patient-years in the bria- kinumab group and 2.7 per 100 patient-years in the methotrexate group.

Cancer was diagnosed in three patients in the briakinumab group (breast cancer in one patient, breast neoplasm [intraductal carcinoma] in one, and prostate cancer in one, at 276, 184, and 205 days, respectively, after the commencement of therapy). One death was reported: a patient in the methotrexate group died from an esophageal rupture. There were no reports of major cardiovascular events such as myocardial infarction, stroke, or death from cardiovascular causes.

Of the 317 patients who took part in the 52week study, 131 in the methotrexate group and 117 in the briakinumab group enrolled in the open-label continuation study. Efficacy results from the open-label continuation study are shown in Table 2 in the Supplementary Appendix. To date, among patients receiving briakinumab continuously in both studies, the adverse-event profile in the open-label continuation study is similar to that in the 52-week trial (Table 3 in the Supplementary Appendix). The adverse-event profile was similar in patients receiving methotrexate during the 52-week trial and patients receiving briakinumab continuously in both the 52-week trial and the open-label continuation study. A total of 154 patients have been treated with briakinumab in the two studies, representing a total of 247.6 patientyears of exposure to briakinumab. Among the patients receiving briakinumab in both trials combined, serious adverse events have been reported in $13.6 \%$ (11.3 events per 100 patient-years), serious infections in $3.2 \%$ ( 2.8 events per 100 personyears), and cancers in $3.2 \%$ (2.0 events per 100 person-years). Nine new serious adverse events have been reported among these patients during the open-label continuation study: one event each of ulcerative colitis, cyst, pyelonephritis, femur fracture, radius fracture, squamous-cell carcinoma, transitional-cell carcinoma, deep-vein thrombosis, and peripheral-artery occlusive disease.

\section{DISCUSSION}

In this 52-week study, briakinumab showed significantly higher efficacy than methotrexate in reducing the signs and symptoms of moderate-to-severe plaque psoriasis. There was a nonsignificant imbalance in some adverse events; serious adverse events, adverse events leading to withdrawal from the study, serious infections, and cancers occurred more fre- 


\begin{tabular}{|c|c|c|c|c|}
\hline \multirow[t]{2}{*}{ Event } & \multicolumn{2}{|c|}{$\begin{array}{c}\text { Briakinumab } \\
\text { ( } \mathrm{N}=154 ; 147.3 \text { Person-Years) }\end{array}$} & \multicolumn{2}{|c|}{$\begin{array}{c}\text { Methotrexate } \\
\text { (N=163; 113.0 Person-Years) }\end{array}$} \\
\hline & no. of patients (\%) & $\begin{array}{c}\text { no. of events } \\
\text { (events/100 person-years) }\end{array}$ & no. of patients (\%) & $\begin{array}{c}\text { no. of events } \\
\text { (events/100 person-years) }\end{array}$ \\
\hline Any adverse event & $131(85.1)$ & $633(429.7)$ & $145(89.0)$ & $555(491.2)$ \\
\hline Serious adverse event $\dagger$ & $14(9.1)$ & 19 (12.9) & $10(6.1)$ & $12(10.6)$ \\
\hline Adverse event leading to discontinuation & $12(7.8)$ & $17(11.5)$ & $10(6.1)$ & $12(10.6)$ \\
\hline \multicolumn{5}{|l|}{ Common adverse eventst } \\
\hline Nasopharyngitis & $44(28.6)$ & $62(42.1)$ & $45(27.6)$ & $69(61.1)$ \\
\hline Headache & $18(11.7)$ & $30(20.4)$ & $22(13.5)$ & $34(30.1)$ \\
\hline Diarrhea & $15(9.7) \int$ & $19(12.9)$ & $6(3.7)$ & $6(5.3)$ \\
\hline Arthralgia & $12(7.8)$ & $16(10.9)$ & $11(6.7)$ & $14(12.4)$ \\
\hline Upper respiratory tract infection & $11(7.1)$ & $18(12.2)$ & $12(7.4)$ & $18(15.9)$ \\
\hline Back pain & $10(6.5)$ & $16(10.9)$ & $9(5.5)$ & $10(8.9)$ \\
\hline Fatigue & $10(6.5)$ & $12(8.1)$ & $10(6.1)$ & $11(9.7)$ \\
\hline Gastroenteritis & $10(6.5)$ & $13(8.8)$ & $11(6.7)$ & $11(9.7)$ \\
\hline Cough & $9(5.8)$ & $11(7.5)$ & $12(7.4)$ & $14(12.4)$ \\
\hline Nausea & $9(5.8)$ & $25(17.0)$ & $19(11.7)$ & $19(16.8)$ \\
\hline Influenza & $8(5.2)$ & $10(6.8)$ & $9(5.5)$ & $10(8.9)$ \\
\hline Rhinitis & $8(5.2)$ & $9(6.1)$ & $6(3.7)$ & $6(5.3)$ \\
\hline \multicolumn{5}{|l|}{ Adverse events of special interest } \\
\hline Injection-site-related event & $13(8.4) 9$ & $27(18.3)$ & $3(1.8)$ & $3(2.7)$ \\
\hline Infection & $89(57.8)$ & $197(133.7)$ & $102(62.6)$ & $185(163.7)$ \\
\hline Serious infection & $4(2.6)$ & $6(4.1)$ & $3(1.8)$ & $3(2.7)$ \\
\hline Opportunistic infection & $1(0.6)$ & $1(0.7)$ & 0 & 0 \\
\hline Cancer & $3(1.9)$ & $3(2.0)$ & 0 & 0 \\
\hline Liver-related event & $10(6.5)$ & $16(10.9)$ & $16(9.8)$ & $29(25.7)$ \\
\hline Cardiovascular event $\|$ & 0 & 0 & 0 & 0 \\
\hline Ischemic heart disease & $6(3.9)$ & $6(4.1)$ & $4(2.5)$ & $5(4.4)$ \\
\hline Death & 0 & 0 & $1(0.6) * *$ & $1(0.9)$ \\
\hline
\end{tabular}

* The events included are those that occurred at the time the first dose of the study drug was administered or any time thereafter, up to 45 days after the last dose was administered.

$\dagger$ The serious adverse events were as follows: in the briakinumab group, two events of fistula and one event each of septic shock, gastrointestinal hypomotility, legionella infection, candidiasis, ankle fracture, intermittent claudication, constipation, breast cancer, osteomyelitis, prostate cancer, breast neoplasm, herpes zoster, tonsillitis, hyperthyroidism, intervertebral-disk protrusion, jaw fracture, and anaphylactoid reaction; and in the methotrexate group, two events of diverticulitis and one event each of increased hepatic-enzyme levels, hepatitis, esophageal rupture, intestinal polyp, sacroiliitis, erythrodermic psoriasis, vertigo, angioedema, urticaria, and intermittent claudication.

+ Included are events that occurred in at least $5 \%$ of patients in either treatment group.

$\int \mathrm{P}=0.04$ for the comparison with methotrexate.

9 $\mathrm{P}=0.009$ for the comparison with methotrexate.

$\|$ Included were any serious adverse events involving myocardial infarction, stroke, or death from cardiovascular causes.

**: The death was due to esophageal rupture.

quently among patients receiving briakinumab than methotrexate group, the percentage of patients among those receiving methotrexate (Table 2).

This study provides insight into the maintenance of a response with methotrexate. In the ing the selection of week 24 as an appropriate time with at least $75 \%$ improvement in the PASI score was the highest between weeks 16 and 24, support- 
point for the assessment of efficacy. After week 24 , there was a relatively sharp drop in the percentage of patients in that group who had at least $75 \%$ improvement in the PASI score. It should be noted that at week 24 , patients who did not have both an improvement of at least $75 \%$ in the PASI score and a score of 0 or 1 on the physician's global assessment discontinued the study per protocol; the largest decrease in the percentage of patients remaining in the study occurred between weeks 24 and 28, with more patients in the methotrexate group than in the briakinumab group discontinuing the study owing to a lack of efficacy. The rates of response with respect to both the PASI and the physician's global assessment were calculated in a conservative fashion by imputing nonresponses for patients who discontinued the study.

With respect to the long-term safety of methotrexate, in previous, 16-week trials, hepatic adverse events led to discontinuation in 12 of 43 patients (28\%) who received $15 \mathrm{mg}$ per week $\mathrm{k}^{22}$ and in 4 of 110 patients (4\%) who received a starting dose of $7.5 \mathrm{mg}$ per week, which was increased to $25 \mathrm{mg}$ per week if required and if the side-effect profile warranted it. ${ }^{23}$ In the present, 52-week study, in which methotrexate was administered at a dose of 5 to $25 \mathrm{mg}$ per week, 16 patients $(9.8 \%)$ in the methotrexate group had liver-related adverse events, but only 2 of these patients $(1.2 \%$ of all the patients in the methotrexate group) discontinued the study drug. These results suggest that initiating methotrexate at a low dose and increasing it as indicated and as tolerated may be a safe strategy for long-term use of this drug.
The incidence of serious infections was 4.1 events per 100 patient-years with briakinumab and 2.7 events per 100 patient-years with methotrexate. Although more patients in the briakinumab group than in the methotrexate group had serious infections and cancers, too few patients were enrolled in the study to draw conclusions regarding these adverse events. No serious cardiovascular events (e.g., myocardial infarction and stroke) were reported during this study, in contrast with low frequencies of such events in another trial of briakinumab $^{13}$ and in trials of ustekinumab. ${ }^{11,12,24}$

One of the limitations of this study was the lack of evaluation of manifestations of psoriasis, such as psoriatic arthritis. A recent study showed that ustekinumab was effective in reducing the signs and symptoms of psoriatic arthritis ${ }^{25}$; future studies are needed to evaluate the effects of briakinumab in patients with psoriatic arthritis.

In conclusion, in patients with moderate-tosevere psoriasis, briakinumab showed higher efficacy than methotrexate through 52 weeks of treatment. Serious adverse events, serious infections, and cancers occurred more frequently among patients receiving briakinumab than among those receiving methotrexate, but the differences were not significant.

Supported by Abbott Laboratories.

Disclosure forms provided by the authors are available with the full text of this article at NEJM.org.

We thank Amy Gamelli, Ph.D., of Abbott Laboratories and Robin L. Stromberg, Ph.D., of Arbor Communications for assistance with the writing of the manuscript, and Michele L. Olds of Abbott Laboratories for assistance with the statistical analyses.

REFERENCES

1. Krueger JG, Bowcock A. Psoriasis pathophysiology: current concepts of pathogenesis. Ann Rheum Dis 2005;64: Suppl 2:ii30-ii36.

2. Nestle FO, Kaplan DH, Barker J. Psoriasis. N Engl J Med 2009;361:496-509.

3. Finlay AY, Coles EC. The effect of severe psoriasis on the quality of life of 369 patients. Br J Dermatol 1995;132:236-44.

4. Krueger G, Koo J, Lebwohl M, Menter A, Stern RS, Rolstad T. The impact of psoriasis on quality of life: results of a 1998 National Psoriasis Foundation patientmembership survey. Arch Dermatol 2001; 137:280-4.

5. Lee E, Trepicchio WL, Oestreicher JL, et al. Increased expression of interleukin 23 p19 and p40 in lesional skin of patients with psoriasis vulgaris. J Exp Med 2004; 199:125-30.
6. Piskin G, Sylva-Steenland RM, Bos JD, Teunissen MB. In vitro and in situ expression of IL-23 by keratinocytes in healthy skin and psoriasis lesions: enhanced expression in psoriatic skin. J Immunol 2006;176:1908-15.

7. Yawalkar N, Karlen S, Hunger $R$, Brand CU, Braathen LR. Expression of interleukin-12 is increased in psoriatic skin. J Invest Dermatol 1998;111:1053-7.

8. Capon F, Di Meglio P, Szaub J, et al. Sequence variants in the genes for the interleukin-23 receptor (IL23R) and its ligand (IL12B) confer protection against psoriasis. Hum Genet 2007;122:201-6.

9. Cargill M, Schrodi SJ, Chang M, et al. A large-scale genetic association study confirms IL12B and leads to the identification of IL23R as psoriasis-risk genes. Am J Hum Genet 2007;80:273-90.
10. Nair RP, Duffin KC, Helms C, et al. Genome-wide scan reveals association of psoriasis with IL-23 and NF-kappaB pathways. Nat Genet 2009;41:199-204.

11. Leonardi CL, Kimball AB, Papp KA, et al. Efficacy and safety of ustekinumab, a human interleukin-12/23 monoclonal antibody, in patients with psoriasis: 76week results from a randomised, doubleblind, placebo-controlled trial (PHOENIX 1). Lancet 2008;371:1665-74. [Erratum, Lancet 2008;371:1838.]

12. Papp KA, Langley RG, Lebwohl M, et al. Efficacy and safety of ustekinumab, a human interleukin-12/23 monoclonal antibody, in patients with psoriasis: 52-week results from a randomised, double-blind, placebo-controlled trial (PHOENIX 2). Lancet 2008;371:1675-84.

13. Gordon K, Langley RG, Gottlieb AB, 
et al. Efficacy and safety results from a phase III, randomized controlled trial comparing two dosing regimens of ABT874 to placebo in patients with moderate to severe psoriasis. Presented at the 3rd International Congress of Psoriasis, Paris, July 1-4, 2010. abstract.

14. Kimball $\mathrm{AB}$, Gordon $\mathrm{KB}$, Langley RG, Menter A, Chartash EK, Valdes J. Safety and efficacy of ABT-874, a fully human interleukin 12/23 monoclonal antibody, in the treatment of moderate to severe chronic plaque psoriasis: results of a randomized, placebo-controlled, phase 2 trial. Arch Dermatol 2008;144:200-7.

15. Schmitt J, Zhang Z, Wozel G, Meurer M, Kirch W. Efficacy and tolerability of biologic and nonbiologic systemic treatments for moderate-to-severe psoriasis: meta-analysis of randomized controlled trials. Br J Dermatol 2008;159:513-26.

16. Menter A, Korman NJ, Elmets CA, et al. Guidelines of care for the management of psoriasis and psoriatic arthritis: sec- tion 4: guidelines of care for the management and treatment of psoriasis with traditional systemic agents. J Am Acad Dermatol 2009;61:451-85.

17. Ko H-S. Clinical trial design in psoriasis. Presented at the 49th Meeting of the Dermatologic and Ophthalmologic Advisory Committee, Bethesda, MD, March 20, 1998. abstract.

18. Rich P, Scher RK. Nail Psoriasis Severity Index: a useful tool for evaluation of nail psoriasis. J Am Acad Dermatol 2003;49:206-12.

19. Finlay AY, Khan GK. Dermatology Life Quality Index (DLQI) - a simple practical measure for routine clinical use. Clin Exp Dermatol 1994;19:210-6.

20. Hongbo Y, Thomas CL, Harrison MA, Salek MS, Finlay AY. Translating the science of quality of life into practice: what do Dermatology Life Quality Index scores mean? J Invest Dermatol 2005;125:659-64 21. Khilji FA, Gonzalez M, Finlay AY. Clinical meaning of change in Dermatol- ogy Life Quality Index scores. Br J Dermatol 2002;147:Suppl 62:50.

22. Heydendael VM, Spuls PI, Opmeer BC, et al. Methotrexate versus cyclosporine in moderate-to-severe chronic plaque psoriasis. N Engl J Med 2003;349:658-65.

23. Saurat JH, Stingl G, Dubertret L, et al. Efficacy and safety results from the randomized controlled comparative study of adalimumab vs. methotrexate vs. placebo in patients with psoriasis (CHAMPION). Br J Dermatol 2008;158:558-66.

24. Krueger GG, Langley RG, Leonardi C, et al. A human interleukin-12/23 monoclonal antibody for the treatment of psoriasis. N Engl J Med 2007;356:580-92.

25. Gottlieb A, Menter A, Mendelsohn A, et al. Ustekinumab, a human interleukin 12/23 monoclonal antibody, for psoriatic arthritis: randomised, double-blind, placebo-controlled, crossover trial. Lancet 2009;373:633-40. [Errata, Lancet 2009; 373:1340, 2010;376:1542.]

Copyright () 2011 Massachusetts Medical Society.

AN NEJM APP FOR IPHONE

The NEJM Image Challenge app brings a popular online feature to the smartphone. Optimized for viewing on the iPhone and iPod Touch, the Image Challenge app lets you test your diagnostic skills anytime, anywhere. The Image Challenge app randomly selects from 300 challenging clinical photos published in NEJM, with a new image added each week. View an image, choose your answer, get immediate feedback, and see how others answered.

The Image Challenge app is available at the iTunes App Store. 